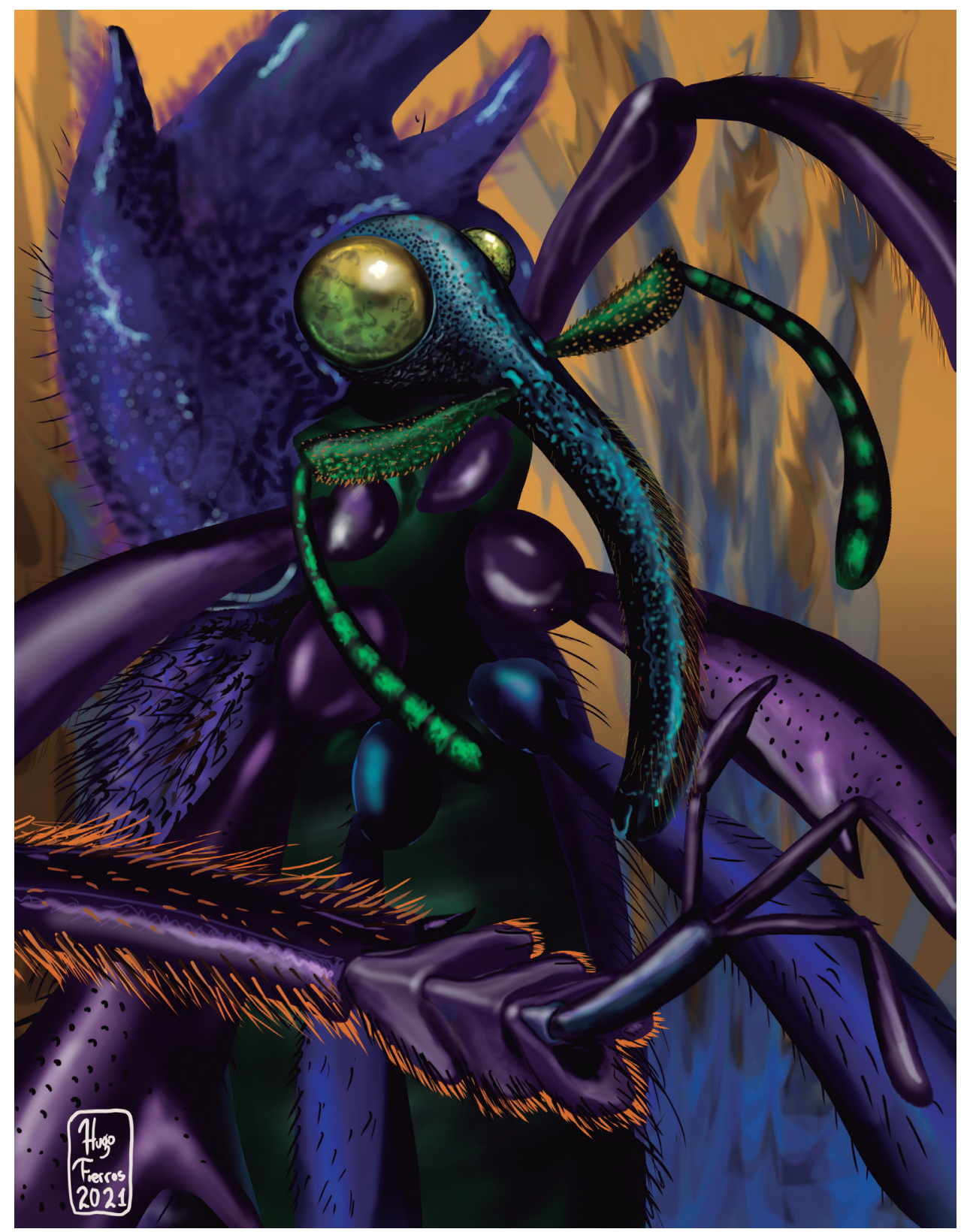

Dugesiana, Año 28, No. 2, (julio-diciembre, segundo semestre de 2021), es una publicación semestral, editada por la Universidad de Guadalajara, a través del Centro de Estudios en Zoología, por el Centro Universitario de Ciencias Biológicas y Agropecuarias. Camino Ramón Padilla Sánchez \# 2100, Nextipac, Zapopan, Jalisco, Tel. 37771150 ext. 33218, http://148.202.248.171/dugesiana/index.php/DUG/index, glenusmx@gmail.com. Editor responsable: José Luis Navarrete-Heredia. Reserva de Derechos al Uso Exclusivo 04-2009-062310115100-203, ISSN: 2007-9133, otorgados por el Instituto Nacional del Derecho de Autor. Responsable de la última actualización de este número: José Luis Navarrete-Heredia, Editor y Ana Laura González-Hernández, Asistente Editorial. Fecha de la última modificación 1 de julio de 2021, con un tiraje de un ejemplar.

Las opiniones expresadas por los autores no necesariamente reflejan la postura del editor de la publicación.

Queda estrictamente prohibida la reproducción total o parcial de los contenidos e imágenes de la publicación sin previa autorización de la Universidad de Guadalajara. 


\title{
Riqueza y Endemismo de Papilionoidea (Lepidoptera) de la región Loxicha en el estado de Oaxaca, México
}

\author{
Richness and Endemism of the Papilionoidea (Lepidoptera) of the Loxicha region, state of Oaxaca, \\ Mexico
}

\section{Armando Luis-Martínez ${ }^{1,2}$, Omar Ávalos-Hernández ${ }^{1,3}$, Marysol Trujano-Ortega ${ }^{1,4 *}$, Arturo Arellano-Covarrubias ${ }^{1,5}$, Isabel Vargas-Fernández ${ }^{1,6}$ y Jorge Llorente-Bousquets ${ }^{1,7}$.}

${ }^{1}$ Museo de Zoología (Entomología), Departamento de Biología Evolutiva, Facultad de Ciencias, Universidad Nacional Autónoma de México, 04510, Ciudad de México, México. *Autor de correspondencia: marysol_trujano@yahoo.com.mx; 2alm@ciencias.unam.mx, ORCID: https://orcid.org/00000002-1044-3986; 30maravalosh@ciencias.unam.mx, ORCID: https:/orcid.org/0000-0002-5476-9400; 4marysol_trujano@yahoo.com.mx, https://orcid.org/0000-0001-8911-8504; 5arellano.covarrubias@ gmail.com, ORCID: https://orcid.org/0000-0001-9515-5782; ${ }^{1}$ ivf@ciencias.unam.mx, ORCID: https:// orcid.org/0000-0001-6524-7184; ${ }^{7}$ enantia@prodigy.net.mx, ORCID: https://orcid.org/0000-0003-08760533 .

\section{RESUMEN}

La región Loxicha en el sur de Oaxaca, México, presenta gran riqueza específica y endemismo de mariposas. Se realizó un estudio faunístico sistemático que incluyó siete años de recolecta durante el período 2005 a 2014. Se describe la diversidad de cuatro familias de Papilionoidea en la región Loxicha, respecto a la riqueza y endemismo del estado y del sur del país. Además, se presenta la lista de especies con 310 taxones de los cuales el 28\% tiene algún grado de endemismo (región, estado, país). La diversidad de Loxicha se compara con la de Sierra de Atoyac en Guerrero, ambas áreas en la Sierra Madre del Sur en la vertiente del Pacífico que en conjunto registran 335 especies, 99 de ellas endémicas al país. La proporción de endemismos de mariposas en estas localidades en la vertiente del Pacífico es mayor en comparación con la Sierra de Juárez en la vertiente del Golfo, la cual es más rica en especies; ello muestra la distinción en los patrones espaciales de riqueza y endemismo de estos insectos. Se discute la importancia de los estudios faunísticos con esfuerzo de muestreo suficiente en el conocimiento y monitoreo de la biodiversidad. La estrategia empleada para Papilionoidea en la región Loxicha puede replicarse en otras regiones y otros grupos megadiversos para obtener listas de especies más completas. Este estudio es parte de un proyecto más amplio que busca conocer los patrones de diversidad y biogeográficos de las mariposas en México.

Palabras claves: lista de especies, faunística, esfuerzo de muestreo, mariposas diurnas, Sierra Madre del Sur.

\section{ABSTRACT}

The Loxicha region in the south of Oaxaca, Mexico has great species richness and endemism of butterflies. We made a systematic faunistic study including sampling in seven years from 2005 to 2014 . We describe the diversity of four families of Papilionoidea in the Loxicha Region, with respect to the species richness and endemism of the state and the south of México. We also present a list of species. We recorded 310 taxa from which $28 \%$ is endemic to some degree (region, state, country). We compare the diversity of the Loxicha Region to that in Sierra de Atoyac, both regions within the Sierra Madre del Sur in the Pacific slope, both regions combined contain 335 species from which 99 are endemic. The endemism ratio of butterflies in these two localities in the Pacific Slope is higher compared to Sierra de Juarez in the Gulf Slope, which has higher species richness, showing the differences between richness and endemism spatial patterns of these insects. We discuss the importance of faunistic analyses with sufficient sampling effort on the understanding and monitoring of biodiversity. The strategy applied for the Papilionoidea in the Loxicha region can be replicated in other regions with other megadiverse groups to obtain the complete lists of species. This research is part of a wider project focused on the study of the diversity and biogeographical patterns of butterflies in Mexico.

Key words: list of species, faunistic, sampling effort, butterflies, Sierra Madre del Sur.

En el segundo lustro de este siglo se inició el proyecto "Las mariposas del estado de Oaxaca", cuyo objetivo principal ha sido estudiar la riqueza específica y la distribución espacial y temporal de la superfamilia Papilionoidea (sensu lato) a través de muestreos exhaustivos y sistemáticos; la revisión de Luis-Martínez et al. (2004) fue el punto de partida de esta serie de investigaciones. Así, desde 2005 se censaron diferentes regiones geográficas de Oaxaca, mediante trabajos faunísticos en sitios de gran diversidad, con el propósito principal de actualizar la lista de Papilionoidea (v. gr. valle de Cuicatlán y región Mixe). A menudo, se abarcaron transectos altitudinales-vegetacionales para conocer la distribución local y fenología de la lepidopterofauna, en especial de los taxones endémicos de Oaxaca y México (Luis-Martínez et al. 2016).

Luis-Martínez et al. (2016) mencionan que Oaxaca es el estado más rico y endémico del país, con 1331 taxones a nivel específico, que pertenecen a 462 géneros, 24 sub- 
familias y seis familias de Papilionoidea; que constituyen el $68 \%$ de los taxones registrados para México de acuerdo con los datos de Llorente-Bousquets et al. (2014). Además, señalan que 259 especies y subespecies son endémicas de México, lo que representa el $19.5 \%$ del total en el estado y $57.5 \%$ de los endémicos que ocurren en el país.

El endemismo de Papilionoidea de México se manifiesta a diferentes niveles taxonómicos, por lo que hay decenas de géneros que se diversificaron en el país. Algunos de estos géneros endémicos son monotípicos y posiblemente paleoendémicos relictos (Llorente-Bousquets et al. 2014), que se distribuyen principalmente en la Sierra Madre del Sur en Guerrero y Oaxaca, así como en la vertiente del Pacífico. Como ejemplos están Baronia Salvin, 1893, Prestonia Schaus, 1920 y Eucheira Westwood, 1834 que son uniespecíficos y están presentes en la región Loxicha. Esta región en la Sierra Madre del Sur ha sido reconocida por varios autores como un centro de endemismo para las mariposas diurnas y otros taxones, especialmente en el intervalo altitudinal de los 800-1400 m (v. gr. Llorente-Bousquets 1983; Llorente-Bousquets y Escalante-Pliego 1992; Llorente-Bousquets et al. 2014; Trujano-Ortega et al. 2015; Luis-Martínez et al. 2016).

La región Loxicha se ubica al sur de Oaxaca y forma parte de las provincias biogeográficas Sierra Madre del Sur y Llanura Costera del Pacífico. Esta región fue objeto de recolectas esporádicas de Papilionoidea durante las últimas cinco décadas del siglo XX; lo que culminó en la descripción de una docena de taxones endémicos a dicha área, a la Sierra Madre del Sur, a la vertiente del Pacífico y/o a México (v. gr. Llorente-Bousquets et al. 2013).

La tasa de destrucción de los hábitats en México se ha acelerado (Dirzo et al. 2009; Torres-Rojo et al. 2016), lo que justifica realizar inventarios bióticos en muchas áreas del país. A través de los inventarios se reconocen zonas con riqueza de especies y/o grado de endemismo notables, las cuales deben considerarse en las estrategias de protección en diversas regiones bióticas (Raguso y Llorente-Bousquets 1991). Conocer la diversidad alfa contenida en una región es importante para la biología de la conservación, ecología y biogeografía; así como describir dicha diversidad en términos altitudinales, fisiográficos, por tipos de vegetación o unidades biogeográficas. No obstante, también son importantes los estudios de distribución local, por microhábitat y a escala fina, para precisar la selección de sitios a conservar y sitios para el aprovechamiento de los recursos biológicos (Arellano-Covarrubias et al. 2018).

El estudio de Papilionoidea adquiere relevancia porque son indicadores de biodiversidad y de modificación de los ecosistemas (Sparrow et al. 1994; Beccaloni y Gaston 1995; Kocher y Williams 2000; Sreekumar y Balakrishnan 2001; Viloria 2002; Tangah et al. 2004; Monteagudo et al. 2001, 2013; Pozo et al. 2015). De esta forma este taxón constituye un modelo de uso frecuente en la elaboración de inventarios faunísticos, por ser un grupo muy diverso, con gran información sobre su historia natural, de captura e identificación relativamente fáciles, representatividad ecológica alta y gran importancia funcional dentro del ecosistema (Luis y Llorente 1990; Brown 1991; Luis-Martínez et al. 1991; Vargas-Fernández et al. 1994, 1999; Moreno 2001; Álvarez et al. 2006; Halffter y Moreno 2005; Llorente-Bousquets et al. 2006; Luis-Martínez et al. 2006; Oñate et al. 2006; Vargas-Fernández et al. 2006). Todo ello le permite ser utilizado para el reconocimiento de áreas de gran diversidad y endemismo, lo que es fundamental en un país con tasas de deforestación en aumento.

Este trabajo presenta la lista de especies de las familias Papilionidae, Pieridae, Nymphalidae y Riodinidae de la región Loxicha en Oaxaca. Se describe la riqueza de mariposas en la zona, que representa la fauna presente a lo largo de un gradiente altitudinal amplio y cinco tipos de vegetación; se reconoce el endemismo a diferentes escalas. Los resultados de este estudio son parte de un proyecto más amplio cuyo objetivo principal es describir los patrones de distribución geográfica de las especies de Papilionoidea en Oaxaca (v. gr. Luis-Martínez et al. 2016; Arellano-Covarrubias et al. 2018; Luis-Martínez et al. 2020). Los especímenes obtenidos durante este trabajo representan muestras para la descripción de especies inéditas, así como para la revisión taxonómica con evidencia morfológica y molecular de algunos taxones (Callaghan et al. 2011, Llorente-Bousquets et al. 2014; Arellano-Covarrubias et al. 2019; Trujano-Ortega et al. 2021). Un análisis detallado de la distribución altitudinal y por tipo de vegetación de Nymphalidae en Loxicha se encuentra en desarrollo; este es equivalente a los publicados recientemente para Riodinidae (Arellano-Covarrubias et al. 2018) y Papilionidae y Pieridae (Luis-Martínez et al. 2020).

\section{MATERIAL Y MÉTODO}

Sitios de muestreo: El muestreo sistemático en la región Loxicha comenzó en 2005, el esfuerzo de muestreo consistió en 229 días de recolección a lo largo de siete años (2005, 2007, 2008, 2011-2014) en 17 sitios de siete municipios en la Sierra Madre del Sur en la vertiente del Pacífico y su planicie costera. Se realizaron muestreos sistemáticos en 13 de los 17 sitios con esfuerzo de muestreo de 10 o más días a lo largo del año; con al menos cinco recolectores por día. Estos sitios se describen en Luis-Martínez et al. (2020) y se ubican geográficamente en Arellano-Covarrubias et al. (2018).

Los sitios se agruparon en tres niveles altitudinales: 0-750, 750-1800, 1800-2850 m y cinco tipos de vegetación: bosque tropical caducifolio, bosque tropical subcaducifolio, bosque mesófilo de montaña, bosque de pino-encino con elementos de bosque mesófilo de montaña y bosque de pino-encino (Arellano-Covarrubias et al. 2018; Luis-Martínez et al. 2020).

Determinación taxonómica: La identificación de las especies se realizó mediante comparación con los especímenes de la Colección de Lepidoptera del Museo de Zoología de la Facultad de Ciencias, UNAM (MZFC). El ordenamiento taxonómico sigue a Lamas (2004), Llorente-Bousquets et al. (2006), Vargas-Fernández et al. (2016), Seraphim et al. (2018) y Trujano-Ortega et al. (2021). Lycaenidae es una familia muy diversa cuya sistemática aún merece atención, por lo que no se incluye aún en este estudio.

Los especímenes se recolectaron bajo el permiso de re- 
colección científica FAUT-0148 emitido por la Secretaría de Medio Ambiente y Recursos Naturales (SEMARNAT). Todos los ejemplares se depositaron en la Colección Lepidoptera (MZFC), registrada en la Secretaría de Medio Ambiente, Recursos Naturales y Pesca (SEMARNAP DFE. IN.071.0798). Los datos recolectados se capturaron en la base de datos MARIPOSA (Luis-Martínez et al. 2005).

\section{RESULTADOS}

Lista de especies. Con las observaciones del trabajo de campo y los registros históricos, la lista de especies de Papilionoidea de la región Loxicha contiene 310 especies en 141 géneros, con 18 subfamilias de cuatro familias de Papilionoidea: Papilionidae, Pieridae, Nymphalidae y Riodinidae (Cuadro 1), que representan el $49 \%$ de la riqueza de Oaxaca. Se obtuvieron 51 registros nuevos de la familia Nymphalidae para la región; estos se señalan en la lista con un asterisco. Pieridae es la familia con mayor representación proporcional al estado (62\%); mientras que la menor fue Riodinidae (40\%) (Luis-Martínez et al. 2016). A nivel nacional, el porcentaje de representación de Pieridae baja a $38 \%$ y de Riodinidae al 25\%. La mitad de las especies de Papilionidae y Nymphalidae reportadas para Oaxaca se ubican en la región Loxicha, lo que denota su gran riqueza.

Los datos de la literatura y de las colecciones refieren principalmente al intervalo altitudinal de los 400 a $1400 \mathrm{~m}$; mientras que los registros de las especies en este estudio, producto del trabajo de campo, se extienden en todo el transecto altitudinal $(0-2850 \mathrm{~m})$. Hay ocho especies de Pieridae, Nymphalidae y Riodinidae que se registran en la literatura, aunque no se recolectaron durante el trabajo de campo de este estudio (señaladas con el superíndice \# en la lista): Ganyra phaloe tiburtia (Fruhstorfer, 1907) (Pieridae), Adelpha donysa ssp., Pedaliodes dejecta ssp. y Actinote guatemalena guerrerensis J. Maza, 1982 (Nymphalidae), Calephelis velutina (Godman \& Salvin, 1878), Emesis lupina lupina Godman \& Salvin, 1886, Thisbe irenea belides Stichel, 1910 e Hypophylla sudias sudias (Hewitson, [1858]) (Riodinidae).

Al considerar el endemismo específico se registraron 21 especies, pero aumentan a 86 taxones si se consideran las subespecies (negritas en la lista). Éstas se dividieron de acuerdo con su distribución espacial en cuatro categorías (superíndice en la lista de especies): 1, endémicos a Loxicha (6 sspp.); 2, endémicos a la Sierra Madre del Sur (21 sspp.); 3, endémicos a la vertiente del Pacífico (32 sspp.) y; 4 , endémicos a una distribución más amplia considerando las tres primeras categorías (27 sspp.). De las seis especies exclusivas a la región Loxicha, cinco pertenecen a Nymphalidae: Memphis wellingi L. Miller \& J. Miller, 1976; Cyllopsis jacquelineae L. Miller, 1974; Callicore texa loxicha R. G. Maza \& J. Maza, 1983; Chlosyne gaudialis wellingi L. Miller \& Rotger, 1979 y Altinote stratonice oaxaca (J. Miller \& L. Miller, 1979); y la otra es la riodínida Synargis nymphidioides praedictum Llorente, Luis \& Arellano, 2013.

Lista de especies de cuatro familias de Papilionoidea de la región Loxicha, Oaxaca, México. Las especies endémi- cas se marcan en negritas, los superíndices indican la escala espacial a la que son endémicos: 1, Loxicha; 2, Sierra Madre del Sur; 3, vertiente del Pacífico; 4, México (ver texto). \# Especies ausentes en este estudio, pero registradas para la zona en la literatura. * Registros nuevos de la familia Nymphalidae para la región Loxicha.

Familia Papilionidae Latreille, [1802]

Subfamilia Baroniinae Bryk, 1913

Género Baronia Salvin, 1893

1. Baronia brevicornis brevicornis Salvin, $1893^{4}$

Subfamilia Papilioninae Latreille, [1802]

Tribu Leptocircini W. F. Kirby, 1896

Género Protographium Munroe, 1961

2. Protographium agesilaus fortis (Rothschild \& Jordan, 1906) ${ }^{3}$

3. Protographium epidaus fenochionis (Salvin \& Godman, 1868) ${ }^{4}$

4. Protographium philolaus philolaus (Boisduval, 1836)

Género Protesilaus Swainson, [1832]

5. Protesilaus macrosilaus penthesilaus (C. Felder \& R. Felder, 1865)

Género Mimoides Brown, 1991

6. Mimoides aconophos (Gray, [1853]) ${ }^{4}$

7. Mimoides ilus occiduus (Vázquez, 1957) ${ }^{4}$

8. Mimoides phaon phaon (Boisduval, 1836)

Tribu Troidini Talbot, 1939

Género Battus Scopoli, 1777

9. Battus eracon (Godman \& Salvin, 1897) ${ }^{4}$

10. Battus philenor philenor (Linnaeus, 1771)

11. Battus polydamas polydamas (Linnaeus, 1758)

Género Parides Hübner, [1819]

12. Parides erithalion trichopus (Rothschild \& Jordan, 1906) ${ }^{4}$

13. Parides montezuma (Westwood, 1842)

14. Parides photinus (Doubleday, 1844)

Tribu Papilionini Latreille, [1802]

Género Heraclides Hübner, [1819]

15. Heraclides anchisiades idaeus (Fabricius, 1793)

16. Heraclides androgeus reyesorum Vargas, Llorente \& Luis, $2012^{3}$

17. Heraclides astyalus bajaensis (J.W. Brown \& Faulkner, 1992) ${ }^{3}$

18. Heraclides erostratus vazquezae (Beutelspacher, 1986) ${ }^{3}$

19. Heraclides ornythion ssp. ${ }^{3}$

20. Heraclides rogeri pharnaces (Doubleday, 1846)

21. Heraclides rumiko Shiraiwa \& Grishin, 2014

22. Heraclides thoas autocles (Rothschild \& Jordan, 1906)

Género Papilio Linnaeus, 1758

23. Papilio polyxenes asterius Stoll, 1782 
Género Pterourus Scopoli, 1777

24. Pterourus multicaudata multicaudata (W.F. Kirby, 1884)

25. Pterourus pilumnus (Boisduval, 1836)

Género Pyrrhosticta Butler, 1872

26. Pyrrhosticta baroni (Rothschild \& Jordan, 1906) ${ }^{2}$

27. Pyrrhosticta menatius morelius (Rothschild \& Jordan, 1906) ${ }^{4}$

Familia Pieridae Swainson, 1820

Subfamilia Dismorphiinae Schatz, 1886

Tribu Dismorphiini Schatz, 1886

Género Enantia, Hübner, [1819]

28. Enantia mazai diazi Llorente, $1984^{3}$

Género Lieinix Gray, 1832

29. Lieinix nemesis nayaritensis Llorente, $1984^{3}$

Género Dismorphia Hübner, 1816

30. Dismorphia amphione isolda Llorente, $1984^{2}$

Subfamilia Coliadinae Swainson, 1821

Género Eurema Hübner, [1819]

31. Eurema daira (Wallengren, 1860)

32. Eurema albula celata (R. Felder, 1869)

33. Eurema arbela boisduvaliana (C. Felder \& R. Felder, 1865)

34. Eurema mexicana mexicana (Boisduval, 1836)

35. Eurema salome jamapa (Reakirt, 1866)

Género Pyrisitia Butler, 1870

36. Pyrisitia dina westwoodi (Boisduval, 1836)

37. Pyrisitia lisa centralis (Herrich-Schäffer, 1865)

38. Pyrisitia nise nelphe (R. Felder, 1869)

39. Pyrisitia proterpia (Fabricius, 1775)

Género Abaeis Hübner, 1819

40. Abaeis nicippe (Cramer, 1779)

Género Nathalis Boisduval, 1836

41. Nathalis iole Boisduval, 1836

Género Kricogonia Reakirt, 1863

42. Kricogonia lyside (Godart, 1819)

Género Zerene Hübner, 1819

43. Zerene cesonia cesonia (Stoll, 1790)

Género Anteos Hübner, [1819]

44. Anteos clorinde (Godart, [1824])

45. Anteos maerula (Fabricius, 1775)

Género Prestonia Schaus, 1920

46. Prestonia clarki Schaus, $1920^{3}$

Género Rhabdodryas Godman \& Salvin, 1889

47. Rhabdodryas trite ssp.

Género Aphrissa Butler, 1873

48. Aphrissa statira statira (Cramer, 1777)
Género Phoebis Hübner, [1819]

49. Phoebis agarithe agarithe (Boisduval, 1836)

50. Phoebis argante ssp.

51. Phoebis neocypris virgo (Butler, 1870)

52. Phoebis philea philea (Linnaeus, 1763)

53. Phoebis sennae marcellina (Cramer, 1777)

Subfamilia Pierinae Swainson, 1820

Tribu Anthocharidini Scudder, 1889

Género Hesperocharis C. Felder, 1862

54. Hesperocharis costaricensis pasion (Reakirt, [1867])

55. Hesperocharis graphites avivolans (Butler, 1865)

Tribu Pierini Swainson, 1820

Género Glutophrissa Butler, 1887

56. Glutophrissa drusilla tenuis (Lamas, 1981)

Género Leptophobia Butler, 1870

57. Leptophobia aripa elodia (Boisduval, 1836)

Género Ascia Scopoli, 1777

58. Ascia monuste monuste (Linnaeus, 1764)

Género Ganyra Billberg, 1820

59. Ganyra josephina josepha (Salvin \& Godman, 1868)

60. Ganyra phaloe tiburtia (Fruhstorfer, 1907) \#

Género Pieriballia Klots, 1933

61. Pieriballia viardi viardi (Boisduval, 1836)

Género Itaballia Kaye, 1904

62. Itaballia demophile centralis Joicey \& Talbot, 1928

Género Melete Swainson, [1831]

63. Melete lycimnia isandra (Boisduval, 1836)

Género Pereute Herrich-Schäffer, 1867

64. Pereute charops sphocra Draudt, $1931^{2}$

Género Catasticta Butler, 1870

65. Catasticta flisa flisa (Herrich-Schäffer, [1858])

66. Catasticta nimbice nimbice (Boisduval, 1836)

67. Catasticta oaxaca Beutelspacher, $1984^{4}$

68. Catasticta teutila ssp. ${ }^{2}$

Género Eucheira Westwood, 1834

69. Eucheira socialis socialis Westwood, $1834^{4}$

Familia Nymphalidae Rafinesque, 1815

Subfamilia Libytheinae Boisduval, 1833

Género Libytheana Michener, 1943

70. Libytheana carinenta mexicana Michener, 1943

Subfamilia Danainae Boisduval, 1833

Tribu Euploeini Herrich-Schäffer, 1849

Género Anetia Hübner, [1823]

71. Anetia thirza thirza Geyer, [1833]

Género Lycorea Doubleday, [1847]

72. Lycorea halia atergatis Doubleday, [1847]

73. Lycorea ilione albescens (Distant, 1876) 
Tribu Danaini Boisduval, 1833

Género Danaus Kluk, 1780

74. Danaus eresimus montezuma Talbot, 1943

75. Danaus gilippus thersippus (Bates, 1863)*

76. Danaus plexippus plexippus (Linnaeus, 1758) *

Subfamilia Ithomiinae Godman \& Salvin, 1879

Tribu Tithoreini Fox, 1940

Género Aeria Hübner, 1816

77. Aeria eurimedia pacifica Godman \& Salvin, 1879

Género Tithorea Doubleday, 1847

78. Tithorea harmonia hippothous Godman \& Salvin, 1879

79. Tithorea tarricina duenna Bates, 1864

Tribu Melinaeini Clark, 1947

Género Melinaea Hübner, 1816

80. Melinaea lilis flavicans Hoffmann, $1924^{3}$

Tribu Mechanitini Bar, 1878

Género Mechanitis Fabricius, 1807

81. Mechanitis lysimnia utemaia Reakirt, 1866

82. Mechanitis menapis doryssus Bates, 1864

83. Mechanitis polymnia lycidice Bates, 1864

Tribu Oleriini Fox, 1940

Género Oleria Hübner, 1816

84. Oleria paula (Weymer, 1883)

Tribu Dircennini D’Almeida, 1941

Género Dircenna Doubleday, 1847

85. Dircenna klugii klugii (Geyer, 1837)

Género Episcada Godman \& Salvin, 1879

86. Episcada salvinia portilla J. Maza \& Lamas, $1978^{3}$

Género Pteronymia Butler \& H. Druce, 1872

87.Pteronymia artena praedicta J. Maza \& Lamas, 1982 2,* 88. Pteronymia cotytto cotytto (Guérin-Méneville, [1844]) *

89. Pteronymia rufocincta (Salvin, 1869) ${ }^{3}$

Tribu Godyridini D’Almeida, 1941

Género Greta Heming, 1934

90. Greta annette moschion (Godman, 1901) ${ }^{3, *}$

91. Greta morgane morgane (Geyer, 1837) ${ }^{3}$

Subfamilia Charaxinae Guenée, 1865

Tribu Anaeini Reuter, 1896

Género Hypna Hübner, [1819]

92. Hypna clytemnestra mexicana Hall, 1917 *

Género Consul Hübner, [1807]

93. Consul electra electra (Westwood, 1850) *

94. Consul excellens genini (Le Cerf, 1922) *

95. Consul fabius cecrops (Doubleday, [1849])
96. Siderone galanthis ssp. *

Género Phantos

97. Phantos callidryas (R. Felder, 1869)

Género Zaretis Hübner, [1819]

98. Zaretis ellops (Ménétriés, 1855)

Género Anaea Hübner, [1819]

99. Anaea troglodyta aidea (Guérin-Méneville, [1844]) *

Género Fountainea Rydon, 1971

100. Fountainea eurypyle glanzi (Rotger, Escalante \& Coronado, 1965$)^{3}$

101.Fountainea glycerium glycerium (Doubleday, [1849])

102.Fountainea nobilis rayoensis (J. Maza \& Díaz, 1978) ${ }^{3}$

Género Memphis Hübner, [1819]

103.Memphis forreri (Godman \& Salvin, 1884) *

104.Memphis perenna perenna (Godman \& Salvin, [1884])

105.Memphis pithyusa pithyusa (R. Felder, 1869) *

106.Memphis wellingi L. Miller \& J. Miller, $1976^{1}$

Tribu Preponini Rydon, 1971

Género Archaeoprepona Fruhstorfer, 1915

107. Archaeoprepona amphimachus baroni J. Maza, $1982^{2}$

108.Archaeoprepona demophon occidentalis Stoffel \& Descimon, $1974^{3}$

109.Archaeoprepona demophoon mexicana Llorente, Descimon \& K. Johnson, $1993^{3}$

110.Archaeoprepona phaedra ssp. ${ }^{2, *}$

Género Prepona Boisduval, 1836

111.Prepona laertes octavia Fruhstorfer, 1905

112.Prepona brooksiana ibarra Beutelspacher, $1982^{32, *}$

Tribu Morphini Newman, 1834

Género Morpho Fabricius, 1807

113.Morpho polyphemus Westwood, [1850]

114. Morpho helenor guerrerensis Le Moult \& Réal, $1962^{3}$

Tribu Brassolini Boisduval, 1836

Género Caligo Hübner, [1819]

115. Caligo telamonius memnon (C. Felder \& R. Felder, 1867)

116. Caligo uranus Herrich-Schäffer, 1850

Género Opsiphanes Doubleday, [1849]

117. Opsiphanes boisduvallii Doubleday, [1849]

118. Opsiphanes cassina fabricii (Boisduval, 1870)

119. Opsiphanes quiteria quirinus Godman \& Salvin, 1881 *

120.Opsiphanes tamarindi tamarindi C. Felder \& R. Felder, 1861

Subfamilia Satyrinae Boisduval, 1833

Género Manataria W. F. Kirby, [1902]

121. Manataria hercyna maculata (Hopffer, 1874)

Género Oxeoschistus A. Butler, 1867

122. Oxeoschistus hilara ssp. ${ }^{2, *}$

123. Oxeoschistus tauropolis ssp. ${ }^{3}$

Género Pedaliodes A. Butler, 1867

Género Siderone Hübner, [1823] 
124.Pedaliodes dejecta ssp. ${ }^{2, \#}$

Género Cissia Doubleday, 1848

125. Cissia similis (Butler, 1867)

126. Cissia terrestris (Butler, 1867) *

127. Cissia $\mathrm{sp.}$

128. Cissia themis (Butler, 1867)

Género Cyllopsis R. Felder, 1869

129. Cyllopsis clinas (Godman \& Salvin, 1889) 2, *

130. Cyllopsis diazi L. Miller, 1974 4,*

131. Cyllopsis hedemanni hedemanni R. Felder, 1869

132. Cyllopsis jacquelineae L. Miller, 1974 1, *

133. Cyllopsis nayarit (R. L. Chermock, 1947) ${ }^{4}$

134. Cyllopsis pyracmon pyracmon (Butler, 1867) *

135. Cyllopsis suivalenoides L. Miller, 1974

Género Euptychia Hübner, 1818

136. Euptychia fetna Butler, 1870

Género Hermeuptychia Forster, 1964

137.Hermeuptychia hermes (Fabricius, 1775)

Género Megisto Hübner, [1819]

138. Megisto rubricata pseudocleophes L. Miller, $1976^{4, *}$

Género Paramacera A. Butler, 1868

139.Paramacera xicaque rubrosuffusa L. Miller, $1972^{2}$

Género Pindis R. Felder, 1869

140.Pindis squamistriga R. Felder, 1869

Género Taygetis Hübner, [1819]

141.Taygetis kerea Butler, 1869

142. Taygetis mermeria griseomarginata L. Miller, $1978^{3}$

143. Taygetis uncinata Weymer, $1907^{4}$

144.Taygetis virgilia (Cramer, 1776)

145.Taygetis weymeri Draudt, 1912

Género Gyrocheilus A. Butler, 1867

146. Gyrocheilus patrobas patrobas (Hewitson, 1862) 4, *

Subfamilia Apaturinae Boisduval, 1840

Género Asterocampa Röber, 1916

147.Asterocampa idyja argus (Bates, 1864)

Género Doxocopa Hübner, [1819]

148. Doxocopa laure laure (Drury, 1773)

149.Doxocopa pavon theodora (Lucas, 1857)

Subfamilia Biblidinae Boisduval, 1833

Tribu Cyrestini Guenée, 1865

Género Marpesia Hübner, 1818

150.Marpesia chiron marius (Cramer, 1779)

151. Marpesia petreus ssp.

152.Marpesia zerynthia dentigera (Fruhstorfer, 1907)

Tribu Biblidini Boisduval, 1833

Género Biblis Fabricius, 1807
153. Biblis hyperia aganisa Boisduval, 1836 *

Género Mestra Hübner, [1825]

154. Mestra dorcas amymone (Ménétriés, 1857) *

Género Hamadryas Hübner, [1806]

155.Hamadryas amphinome mazai Jenkins, $1983^{3}$

156. Hamadryas atlantis lelaps (Godman \& Salvin, 1883) ${ }^{3, *}$

157. Hamadryas februa ferentina (Godart, [1824])

158. Hamadryas glauconome glauconome (Bates, 1864)

159.Hamadryas guatemalena marmarice (Fruhstorfer, 1916) ${ }^{4}$

Género Eunica Hübner, [1819]

160.Eunica alcmena alcmena (Doubleday, [1847])

161.Eunica monima (Stoll, 1782)

162.Eunica tatila tatila (Herrich-Schäffer, [1855]) *

Género Catonephele Hübner, [1819]

163. Catonephele cortesi R. G. Maza, $1982^{3}$

164. Catonephele numilia immaculata Jenkins, $1985^{2}$

Género Myscelia Doubleday, [1845]

165. Myscelia cyananthe cyananthe $\mathrm{C}$. Felder \& R. Felder, $1867^{4}$

166. Myscelia cyaniris alvaradia R. G. Maza \& Díaz, 1982

167.Myscelia ethusa ethusa (Doyère, [1840]) *

Género Nica Hübner, [1826]

168. Nica flavilla bachiana (R. G. Maza \& J. Maza, 1985) ${ }^{2}$

Género Temenis Hübner, [1819]

169. Temenis laothoe quilapayunia R. G. Maza \& Turrent, $1985^{3}$

Género Bolboneura Godman \& Salvin, 1877

170.Bolboneura sylphis beatrix R. G. Maza, $1985^{3, *}$

Género Epiphile Doubleday, [1845]

171.Epiphile adrasta escalantei Descimon \& Mast, 19794

Género Pyrrhogyra Hübner, [1819]

172.Pyrrhogyra edocla paradisea R. G. Maza \& J. Maza, $1985^{3}$

173.Pyrrhogyra neaerea hypsenor Godman \& Salvin, 1884

Género Diaethria Billberg, 1820

174.Diaethria anna mixteca J. Maza, $1977^{2}$

175.Diaethria astala asteroide R. G. Maza \& R. F. Maza, $1985^{2}$

Género Callicore Hübner, [1819]

176. Callicore texa loxicha R. G. Maza \& J. Maza, $1983^{1}$

Género Cyclogramma Doubleday, [1848]

177. Cyclogramma pandama (Doubleday, [1848])

Género Dynamine Hübner, [1819]

178.Dynamine dyonis Geyer, 1837

179.Dynamine postverta mexicana D'Almeida, 1952

180.Dynamine theseus (C. Felder \& R. Felder, 1861) 
Subfamilia Limenitidinae Behr, 1864

Tribu Limenitidini Behr, 1864

Género Adelpha Hübner, [1819]

181.Adelpha barnesia leucas Fruhstorfer, 1915

182.Adelpha basiloides (Bates, 1865)

183. Adelpha bredowii Geyer, 1837 *

184.Adelpha diocles ssp. ${ }^{2, *}$

185.Adelpha donysa ssp. $2, \#$

186. Adelpha fessonia fessonia (Hewitson, 1847)

187. Adelpha iphicleola iphicleola (Bates, 1864)

188.Adelpha iphiclus iphiclus (Linnaeus, 1758)

189. Adelpha leuceria leuceria (Druce, 1874)

190.Adelpha leucerioides ssp. ${ }^{3}$

191.Adelpha lycorias melanthe (Bates, 1864)

192. Adelpha naxia naxia (C. Felder \& R. Felder, 1867)

193.Adelpha paraena massilia (C. Felder \& R. Felder, 1867)

194.Adelpha phylaca phylaca (Bates, 1866)

195.Adelpha pithys (Bates, 1864)

196.Adelpha serpa celerio (Bates, 1864)

Subfamilia Nymphalinae Rafinesque, 1815

Tribu Coeini Scudder, 1893

Género Historis Hübner, [1819]

197. Historis acheronta acheronta (Fabricius, 1775) *

198. Historis odius dious Lamas, 1995

Género Pycina Doubleday, [1849]

199.Pycina zamba zelys Godman \& Salvin, 1884 *

Tribu Nymphalini Rafinesque, 1815

Género Colobura Billberg, 1820

200. Colobura dirce dirce (Linnaeus, 1758)

Género Smyrna Hübner, [1823]

201.Smyrna blomfildia datis Fruhstorfer, 1908

202.Smyrna karwinskii Geyer, [1833] *

Género Hypanartia Hübner, [1821]

203. Hypanartia dione disjuncta Willmott, J. Hall \& Lamas, 2001

204.Hypanartia godmanii (Bates, 1864)

205.Hypanartia lethe (Fabricius, 1793)

206. Hypanartia trimaculata autumna Willmott, J. Hall \& Lamas, 2001

Género Nymphalis Kluk, 1780

207.Nymphalis antiopa antiopa (Linnaeus, 1758) *

Género Vanessa Fabricius, 1807

208. Vanessa atalanta rubria (Fruhstorfer, 1909) *

209.Vanessa cardui (Linnaeus, 1758) *

210. Vanessa virginiensis (Drury, 1773) *

Tribu Victorinini Scudder, 1893

Género Siproeta Hübner, [1823]

211.Siproeta epaphus epaphus (Latreille, [1813])

212.Siproeta stelenes biplagiata (Fruhstorfer, 1907)

Género Anartia Hübner, [1819]

213.Anartia fatima fatima (Fabricius, 1793)

214.Anartia jatrophae luteipicta Fruhstorfer, 1907 *
Tribu Junoniini Reuter, 1896

Género Junonia Hübner, [1819]

215.Junonia coenia Hübner, [1822]

216.Junonia evarete nigrosuffusa Barnes \& McDunnough, 1916 *

217.Junonia genoveva ssp. *

Tribu Melitaeini Herrich-Schäffer, 1843

Género Chlosyne Butler, 1870

218. Chlosyne cynisca (Godman \& Salvin, 1882) 4 , *

219. Chlosyne erodyle ssp.

220. Chlosyne gaudialis wellingi L. Miller \& Rotger, $1979^{1}$

221. Chlosyne hippodrome hippodrome (Geyer, 1837)

222. Chlosyne janais janais (Drury, 1782)

223. Chlosyne lacinia lacinia (Geyer, 1837)

224. Chlosyne marina marina (Geyer, 1837)

225. Chlosyne melanarge (Bates, 1864)

226. Chlosyne theona theona (Ménétriés, 1855)

Género Microtia H.W. Bates, 1864

227. Microtia elva elva Bates, 1864

Género Phyciodes Hübner, [1819]

228. Phyciodes graphica graphica (R. Felder, 1869) *

229. Phyciodes mylitta thebais Godman \& Salvin, 1878

230.Phyciodes pallescens (R. Felder, 1869) ${ }^{4, *}$

231.Phyciodes phaon phaon (Edwards, 1864)

232.Phyciodes tharos tharos (Drury, 1773) *

Género Tegosa Higgins, 1981

233.Tegosa guatemalena (Bates, 1864)

Género Anthanassa Scudder, 1875

234. Anthanassa ardys ardys (Hewitson, 1864) ${ }^{4}$

235. Anthanassa argentea (Godman \& Salvin, 1882)

236. Anthanassa atronia (Bates, 1866)

237. Anthanassa frisia tulcis (Bates, 1864)

238. Anthanassa nebulosa alexon (Godman \& Salvin, 1889) ${ }^{4}$

239.Anthanassa otanes oaxaca Beutelspacher, 1990 4, *

240.Anthanassa ptolyca amator (Hall, 1929) ${ }^{4}$

241.Anthanassa sitalces cortes (Hall, 1917) ${ }^{4}$

242.Anthanassa texana texana (Edwards, 1863)

Género Eresia Boisduval, 1836

243.Eresia phillyra phillyra Hewitson, 1852

Subfamilia Heliconiinae Swainson, 1822

Tribu Acraeini Boisduval, 1833

Género Altinote Potts, 1943

244.Altinote stratonice oaxaca (J. Miller \& L. Miller, 1979) ${ }^{1}$

Género Actinote Hübner, [1819]

245.Actinote guatemalena guerrerensis J. Maza, 1982 2, \#

Tribu Heliconiini Swainson, 1822

Género Agraulis Boisduval \& Le Conte, [1835]

246. Agraulis vanillae incarnata (Riley, 1926) * 
Género Dione Hübner, [1819]

247.Dione juno huascuma (Reakirt, 1866)

248.Dione moneta poeyii Butler, 1873 *

Género Dryadula Michener, 1942

249.Dryadula phaetusa (Linnaeus, 1758)

Género Dryas Hübner, [1807]

250.Dryas iulia moderata (Riley, 1926)

Género Eueides Hübner, 1816

251.Eueides aliphera gracilis Stichel, 1903

252.Eueides isabella eva (Fabricius, 1793) *

Género Heliconius Kluk, 1780

253. Heliconius charithonia vazquezae W. P. Comstock \&

F. M. Brown, 1950

254.Heliconius erato cruentus Lamas, $1998^{3}$

255.Heliconius hortense Guérin-Méneville, [1844]

256.Heliconius ismenius telchinia Doubleday, 1847 *

Tribu Argynnini Swainson, 1833

Género Euptoieta Doubleday, [1848]

257.Euptoieta claudia daunius (Herbst, 1798) *

258.Euptoieta hegesia meridiania Stichel, 1938

Familia Riodinidae Grote, 1895

Subfamilia Nemeobiinae Bates, 1868

Tribu Euselasiini Kirby, 1871

Género Euselasia Hübner, [1819]

259. Euselasia hieronymi hieronymi (Godman \& Salvin, 1868)

260. Euselasia eubule eubule (R. Felder, 1869)

Subfamilia Riodininae Grote, 1895

Tribu Eurybiini Reuter, 1897

Género Mesosemia Hübner, [1819]

261. Mesosemia lamachus Hewitson, 1857

Género Voltinia Stichel, 1910

262. Voltinia umbra (Boisduval, 1870)

Género Eurybia [Illiger], 1807

263. Eurybia elvina elvina Stichel, 1910

Tribu Nymphidiini Bates, 1859

Género Pseudonymphidia Callaghan, 1985

264.Pseudonymphidia agave agave (Godman \& Salvin, 1886)

Género Thisbe Hübner, [1819]

265.Thisbe irenea belides Stichel, 1910 \#

266.Thisbe lycorias (Hewitson, [1853])

Género Synargis Hübner, [1819]

267. Synargis mycone (Hewitson, 1865)

268.Synargis nymphidioides praedictum Llorente, Luis \& Arellano, $2013^{1}$

Género Hypophylla Boisduval, 1836

269. Hypophylla zeurippa Boisduval, 1836

270. Hypophylla sudias sudias (Hewitson, [1858]) \#
Género Theope Doubleday, 1847

271. Theope pseudopedias J. Hall, 1999

272. Theope barea Godman \& Salvin, 1878

273.Theope villai Beutelspacher, $1981^{2}$

274. Theope virgilius (Fabricius, 1793)

275.Theope eupolis Schaus, 1890

276. Theope publius incompositus J. Hall, 1999

277.Theope bacenis Schaus, 1890

Tribu Calydnini Seraphim, Freitas \& Kaminski, 2018 Género Calydna Doubleday, 1847

278. Calydna venusta venusta Godman \& Salvin, 1886

Tribu Helicopini Reuter, 1897

Género Anteros Hübner, [1819]

279. Anteros carausius carausius Westwood, 1851

Tribu Emesidini Seraphim, Freitas \& Kaminski, 2018

Género Emesis Fabricius, 1807

280. Emesis mandana furor Butler \& H. Druce, 1872

281. Emesis tegula Godman \& Salvin, 1886

282. Emesis vulpina Godman \& Salvin, 1886

283.Emesis poeas Godman, $1901^{3}$

284.Emesis tenedia C. Felder \& R. Felder, 1861

285.Emesis lupina lupina Godman \& Salvin, 1886 \#

Género Curvie Grishin, 2019

286. Curvie emesia (Hewitson, 1867)

Género Apodemia C. Felder \& R. Felder, 1865

287.Apodemia multiplaga Schaus, 1902

288. Apodemia walkeri Godman \& Salvin, 1886

Género Neoapodemia Trujano-Ortega, 2018

289. Neoapodemia zela zela Butler, 1870

Tribu Symmachiini Bates, 1859

Género Mesene Doubleday, 1847

290.Mesene jimena Callaghan, Llorente \& Luis, $2011^{2}$

Tribu Riodinini Grote, 1895

Género Rhetus Swainson [1829]

291. Rhetus arcius beutelspacheri Llorente, $1988^{3}$

Género Notheme Westwood, 1851

292. Notheme erota diadema Stichel, 1910

Género Calephelis Grote \& Robinson, 1869

293. Calephelis sp. 1

294. Calephelis sp. 2

295. Calephelis sp. 3

296. Calephelis sp. 4

297. Calephelis sp. 5

298. Calephelis sp. 6

299. Calephelis sp. 7

300. Calephelis velutina (Godman \& Salvin, 1878) \#

Género Caria Hübner, 1823 
301. Caria ino ino Godmann \& Salvin, $1886^{4}$

302. Caria stillaticia Dyar, $1912^{4}$

303. Caria melino Dyar, $1912^{4}$

Género Baeotis Hübner, [1819]

304. Baeotis zonata zonata R. Felder, 1869

Género Lasaia H. W. Bates, 1868

305. Lasaia sula sula Staudinger, 1888

306. Lasaia agesilas callaina Clench, 1972

307. Lasaia sessilis Schaus, 1890

Género Melanis Hübner, [1819]

308. Melanis pixe pixe (Boisduval, 1836)

309. Melanis cephise cephise (Ménétriés, 1855)

\section{Incertae sedis}

Género Lamphiotes Callaghan, 1982

310. Lamphiotes velazquezi (Beutelspacher, 1976) ${ }^{2}$

Diversidad de la región Loxicha en relación con la Sierra Madre del Sur. La Sierra Madre del Sur en su vertiente del Pacífico (Guerrero y Oaxaca) destaca por su diversidad elevada de especies y endemismos. A diferencia del presente estudio que abarca un transecto altitudinal del nivel del mar hasta los $2850 \mathrm{~m}$ de altitud; el único otro estudio faunístico en la misma unidad fisiográfica es el que realizó Vargas-Fernández et al. (1994) en la Sierra de Atoyac en Guerrero, quienes abarcaron un transecto altitudinal que va de los 300 a 3100 m (Cuadro 2). El esfuerzo de muestreo y la amplitud espacial y temporal hacen del estudio faunístico en Loxicha el de mayor esfuerzo de muestreo exhaustivo y sistemático realizado en México (Arellano-Covarrubias et al. 2018; Luis-Martínez et al. 2020). Entre ambos inventarios se registran 335 especies y 142 géneros en 18 subfamilias de las cuatro familias, por lo que la región Loxicha contiene el $92.5 \%$ de la riqueza mientras que Sierra de Atoyac el 77.6\% (Cuadro 2). La riqueza es mayor en la región Loxicha en 14 de las 18 subfamilias, la diferencia es mayor en las subfamilias Riodininae (15) y Nymphalinae (12) y solo las subfamilias Satyrinae y Dismorphiinae son de riqueza superior en Sierra de Atoyac.

El número de especies-subespecies endémicas a México, registradas entre estos dos transectos, es de 99 taxones; lo que corresponde al $29.6 \%$ del total de la fauna de estas áreas y el $37.9 \%$ de las especies endémicas al país en estas cuatro familias, 86 se encuentran en la región Loxicha y 75 en la Sierra de Atoyac, con el 62.6\% de los endémicos compartidos. Al igual que en el caso de la región Loxicha, se dividieron en cuatro categorías: 1, endémicos a Sierra de Atoyac (4 sspp.); 2, endémicos a la Sierra Madre del Sur (23 sspp.); 3, endémicos a la vertiente del Pacífico (30 sspp.) y; 4 , endémicos con una distribución más amplia, además de considerar las tres primeras categorías (18 sspp.). Las especies endémicas a la Sierra de Atoyac son Papilionidae: Protographium thyastes occidentalis; Nymphalidae: Drucina championi ssp., Eunica malvina almae y Eueides isabella nigricornis. Los datos de las tres primeras categorías son muy similares entre ambas áreas, con diferencia de una a dos especies, siempre mayor en la región Loxicha. En la cuarta categoría, que corresponde a las especies que presentan un área de distribución mayor a las Sierra Madre del Sur y la vertiente del Pacífico, se presenta una diferencia de nueve especies.

En ambas regiones, Nymphalidae es la familia que presenta mayor riqueza y número de endemismos; en contraste con Papilionidae que es la de menor riqueza y Riodinidae la de valores de endemismo más bajos.

\section{DISCUSIÓN}

La evaluación de la diversidad biológica, con la riqueza de especies como uno de sus componentes más significativos, es relevante para la conservación y aprovechamiento de recursos. Una tarea crucial para esta evaluación es verificar con precisión las especies que se encuentran presentes en un área determinada. Pero también es importante la evaluación continua de los registros históricos, lo que permite identificar cambios temporales en la riqueza y composición de especies. Para esto son necesarios estudios faunísticos sistemáticos y completos que aumenten las probabilidades de registrar a todas las especies. A pesar del esfuerzo de muestreo tan amplio, en la región Loxicha no se registraron ocho especies que se habían citado con anterioridad o bien que se encuentran en colecciones. La ausencia de registros de estas especies muy probablemente se explica por aspectos de rareza o extinción local, aunque también por otros factores como la determinación taxonómica errónea de los ejemplares o fotografías, el etiquetado erróneo de los ejemplares originalmente recolectados, cambios nomenclaturales y clasificatorios o datos incompletos que carecen de una revisión adecuada. Un ejemplo son algunas especies registradas para Sonora en el atlas de Stanford y Opler (1993); tales registros se consideran cuestionables y carecen de evidencia; es decir, faltan especímenes o fotografías que los respalden (Bailowitz et al. 2017). Dado que no es posible asegurar la ausencia de estas especies, se mantienen en la lista. Los estudios faunísticos periódicos confirmarán o refutarán la presencia de estos taxones en la región, al identificar los cambios en la composición de especies de la región.

La región Loxicha presenta riqueza y endemismo altos respecto al estado y al país, lo que se puede explicar en parte por el esfuerzo de muestreo temporalmente extenso y número de colectores en comparación con otras regiones poco estudiadas. Evaluar la magnitud de esta diversidad es complicado sin otros estudios faunísticos similares, por lo que el inventario de la Sierra de Atoyac es un punto de referencia adecuado debido al nivel de completitud que presenta (Vargas-Fernández et al. 1994). La región de Loxicha presenta una riqueza de especies mayor que Atoyac; sin embargo, la diferencia está en función de las particularidades del muestreo, la heterogeneidad de los ambientes y los gradientes altitudinales representados. Por ejemplo, en Atoyac no se muestreo el bosque tropical caducifolio ni los primeros 300 metros de altitud; mientras que en Loxicha hay registros para este tipo de vegetación entre los 80 y $600 \mathrm{~m}$; por lo tanto, se obtuvieron tres especies en este ambiente en Loxicha que no fueron registradas en Atoyac: 
Baronia b. brevicornis, Bolboneura sylphis beatrix e Hypna clytemnestra mexicana. La magnitud de la diversidad y del endemismo de la región Loxicha se podrá evaluar cuantitativamente cuando se tengan datos de otras regiones con ambientes y esfuerzo de muestreo similares o equivalentes.

En el área de estudio hay varias especies estenoecas o estenotópicas a los bosques mesófilos (Pereute charops sphocra, Episcada salvinia portilla, Fountainea nobilis rayoensis, Prepona brooksiana ibarra), bosques mixtos de pino-encino y coníferas (Pyrrhosticta baroni, Eucheira s. socialis, Paramacera xicaque rubrosuffusa) o a los bosques tropicales, en especial subcaducifolios y caducifolios $(B a-$ ronia b. brevicornis, Prestonia clarki, Bolboneura sylphis beatrix).

Llorente-Bousquets et al. (2014) mencionan que la representación del endemismo de Papilionoidea difiere de los patrones de su riqueza. Es posible analizarlo si se comparan las dos localidades del Pacífico presentadas en este estudio con una del Atlántico, ya que todas ellas presentan un muestreo aceptable de la riqueza específica de mariposas (Luis-Martínez et al. 1991; Vargas-Fernández et al. 1994; Arellano-Covarrubias et al. 2018; Luis-Martínez et al. 2020). La Sierra de Juárez en su vertiente del Atlántico (100 a los $3100 \mathrm{~m}$ de altitud) presenta 390 especies (Luis-Martínez et al. 1991), por lo que supera en número de especies a la región de Loxicha con 80 especies, a Atoyac con 130 y a Loxicha-Atoyac con 55; lo que coincide con los patrones de riqueza previamente observados para las mariposas, donde la riqueza es mayor en la vertiente atlántica, principalmente en el bosque tropical perennifolio (Ross 1975-1977; Luis-Martínez et al. 1991; Salinas-Gutiérrez et al. 2004; Llorente-Bousquets et al. 2014; Flores-Contreras y Luna-Reyes 2017). Por otro lado, la Sierra de Juárez presenta tan solo 33 especies endémicas en contraste con 86 de la región Loxicha, 75 de Atoyac y 99 de Loxicha-Atoyac; por lo que el endemismo de las cuatro familias de Papilionoidea se concentra en el oeste y sur del país, no en el sureste (Llorente-Bousquets et al. 2014; Luis-Martínez et al. 2016, 2020). El endemismo de la Sierra de Juárez representa el $8.46 \%$ y está muy por debajo de los valores que presentan Loxicha y Atoyac, que exhiben el 27.4 y el $28.46 \%$ respectivamente.

Este ejercicio comparativo muestra una vez más los patrones diferenciales entre la riqueza y el endemismo de Papilionoidea (Llorente-Bousquets et al. 2014), donde es posible que la historia biogeográfica de la Sierra Madre del Sur explique el patrón observado, al producir aislamiento y la presencia de ambientes restringidos, como sucede con las especies exclusivas a la Sierra de Atoyac de Álvarez en Guerrero y a la región Loxicha en Oaxaca (Llorente 1983; Luis-Martínez et al. 1991; Vargas-Fernández et al. 1994; Arellano-Covarrubias et al. 2018; Luis-Martínez et al. 2016, 2020). Es evidente que varios taxones con distribuciones archipelágicas asociados a los bosques mesófilos de montaña tienen su afinidad geográfica con sus taxones hermanos en el Núcleo Montano de América Central (sensu Halffter, 1976) (v. gr. Lieinix neblina, Drucina championi ssp.); mientras que las especies endémicas de los bosques tropicales son más cercanas con los taxones de la vertiente del Atlántico y sureste del país (v. gr. Eueides isabella nigricornis, Callicore texa loxicha).

Los resultados obtenidos para la región Loxicha demuestran cómo un estudio faunístico sistemático incrementa de forma relevante el conocimiento de la fauna de una región. Con un esfuerzo de muestreo adecuado se puede reconocer el valor biológico de una región y su importancia a nivel regional, estatal y nacional. Esto solo es posible con la inversión de recursos para trabajo de campo, colección y personal especializado durante un periodo de tiempo suficiente (Llorente-Bousquets y Ocegueda 2008). La estrategia aquí empleada consiste en concentrarse en un área por un periodo relativamente largo, en contraste con la inclusión de varias áreas con muestreos de menor escala temporal en cada una. Esta estrategia resultó efectiva para conocer la riqueza de Papilionidae, Pieridae, Nymphalidae y Riodinidae de la región Loxicha (Arellano-Covarrubias et al. 2018; Luis-Martínez et al. 2020) y podría emplearse en otros grupos taxonómicos y otras regiones. En particular, para conocer la diversidad de grupos megadiversos de insectos que a menudo solo se conocen de modo parcial en estudios faunísticos de menor escala.

El conocimiento de la diversidad de mariposas de esta región, que comienza con la lista de especies que se presenta, pronto permitirá análisis de la diversidad más elaborados en diferentes unidades ecológicas, temporales y espaciales y con ello el avance taxonómico, ecológico y biogeográfico de este grupo de insectos, ambientes y áreas del país, así como sus consecuencias en bioconservación.

\section{AGRADECIMIENTOS}

Los autores deseamos dedicar este trabajo a nuestro compañero y amigo, el Dr. Juan José Morrone, por su dedicación al entendimiento y comprensión de la historia geográfica de México y América, su labor en el análisis y estandarización de los conceptos biogeográficos y fraguar el estudio espacial y temporal de la diversidad, siempre con un lenguaje 'sencillito'.

Agradecemos a la CONABIO, la cual, en sus tres décadas de existencia, ha apoyado a la colección de Lepidoptera, con recursos para el fortalecimiento de la infraestructura, trabajo de campo y para la repatriación de datos de los ejemplares depositados en las colecciones más importantes de los Estados Unidos de América (1991-2000). Así mismo a los proyectos DGAPA PAPIIT IN212418, PAPIIT-IN220521, PAPIME-PE202820 y CONACyT 284966. MTO agradece el apoyo de CONACyT 284966. A los curadores de las colecciones consultadas para la construcción de la base de datos MARIPOSA, quienes facilitaron el acceso a las colecciones y bibliografía bajo su custodia: Frederick Rindge $\uparrow$ (American Museum of Natural History, Nueva York), Jerry Powell y John Chemsak (Colección "Essig" del Depto. de Ciencias Entomológicas, Universidad de California, campus Berkeley, California), John E. Rawlins (Carnegie Museum of Natural History, Pittsburgh, Pennsylvania), David K. Faulkner y John W. Brown (San Diego Natural History Museum, California), Paul Arnaud Jr. y Norman Penny (California Academy of Sciences, San Francisco, California), Brian V. Brown y Brian Harris 
(Los Angeles County Museum, California), Lee D. Miller † (Museo Allyn de Entomología, Sarasota, Florida), George T. Austin f (Nevada State Museum), Philip Ackery y Dick Vane-Wright (British Museum of Natural History), Harry Brailovsky (Colección Entomológica del Instituto de Biología, UNAM, Cd. de México), Robert K. Robbins (National Museum of Natural History, Smithsonian Institution, Washington, D.C.), Thomas Emmel f, Jacqueline Miller y Andrew D. Warren (Colección del McGuire Center for Lepidoptera and Biodiversity, Florida Museum of Natural History, University of Florida), Carmen Pozo de la Tijera (Colección de Lepidoptera del Colegio de la Frontera Sur, Chetumal) y a Mercedes Luna (Colección de Lepidoptera del Museo de Zoología de la Facultad de Estudios Superiores Zaragoza, UNAM).

También tenemos que agradecer a numerosos habitantes de Oaxaca y propietarios de predios que nos apoyaron en campo, como Armando Canavati (Rancho Hagia Sofía, Santa María Huatulco). Los responsables del área protegida del Parque Nacional Huatulco quienes nos brindaron también su apoyo a lo largo de cinco años. A Jimena Castro por su apoyo en el trabajo de gabinete y a Alejandra Sánchez García, Andrew D. Warren, Blanca Claudia Hernández Mejía, Ela Stephanie Esquivel Ruíz, Jessica Hernández Jerónimo, John Kemner, José Luis Salinas Gutiérrez, Marisol Esther Almaraz Almaraz, Sandra Nieves Uribe y Uri Omar García Vázquez, quienes nos apoyaron en el trabajo de campo. Además de Atzinameyali Sánchez Castañeda, quien preparo en alfiler todo el material de este proyecto para su ingreso en la colección. A José Luis Navarrete-Heredia, Mercedes Luna-Reyes y un revisor anónimo por las sugerencias para enriquecer el trabajo.

\section{LITERATURA CITADA}

Álvarez, M., S. Córdoba, F. Escobar, G. Fagua, F. Gast, H. Mendoza, M. Ospina, A. Umaña, y H. Villarreal. 2006. Insectos. (pp. 149-184). En: Álvarez, M., S. Córdoba, F. Escobar, G. Fagua, F. Gast, H. Mendoza, M. Ospina, A. Umaña y H. Villarreal (Eds.). Manual de métodos para el desarrollo de inventarios de Biodiversidad. Instituto de Investigación de Recursos Hidrológicos Alexander von Humboldt. Bogotá, Colombia.

Arellano-Covarrubias, A., J. Llorente-Bousquets y A. Luis-Martínez. 2018. Distribución y fenología de la familia Riodinidae (Lepidoptera: Papilionoidea) en el bosque tropical subcaducifolio de Oaxaca, México. $R e$ vista de Biología Tropical, 66(2): 503-558.

Arellano-Covarrubias, A., M. Trujano-Ortega, A. Luis-Martínez, M. Luna-Reyes and J. Llorente-Bousquets. 2019. Geographical distribution of Lasaia Bates, 1868 (Lepidoptera: Riodinidae) across the biogeographical provinces of Mexico. Zootaxa, 4656(2): 243-273.

Bailowitz, R., J. Brock and D. Danforth. 2017. Annotated checklist of the butterflies (Lepidoptera) of Sonora, Mexico. Dugesiana, 24(2): 125-147.

Beccaloni, G.W. and K.J. Gaston. 1995. Predicting the species richness of neotropical forest butterflies: Ithomiinae (Lepidoptera: Nymphalidae) as indicators. Biolog- ical Conservation, 71(1): 77-86.

Brown, K.S. Jr. 1991. Conservation of neotropical environments: insects as indicators. (pp. 349-404). In: Collins, N.M. and J.A. Thomas (Eds.). The conservation of insects and their habitats. Academic Press, London, England.

Callaghan, C., J. Llorente-Bousquets and A. Luis-Martínez, A. 2011. A new Mexican Mesene (Lepidoptera, Riodinidae). Zootaxa, 2896(1): 53-64.

Dirzo, R., A, Aguirre y J.C. López. 2009. Diversidad florística de las selvas húmedas en paisajes antropizados. Investigación, 1(1): 17-22.

Halffter, G. y C.E. Moreno. 2005. Significado biológico de las diversidades alfa, beta y gamma. (pp. 5-18). En: Halffter, G., J. Soberón, P. Koleff y A. Melic (Eds.). Sobre Diversidad Biológica: el significado de las Diversidades Alfa, Beta y Gama. m3m: Monografías Tercer Milenio, Vol. 4. Zaragoza, España.

Flores-Contreras, I. y M. Luna-Reyes. 2017. Diversidad y Distribución de cinco familias de Papilionoidea (Lepidoptera) de las Selvas Altas en la Provincia Biogeográfica del Golfo de México. Acta Zoológica (Nueva Serie), 33(2): 211-230.

Kocher, S.D. and E.H. Williams. 2000. The diversity and abundance of North American butterflies vary with habitat disturbance and geography. Journal of Biogeography, 27(4): 785-794.

Lamas, G. 2004. Checklist: Part 4A. Hesperioidea - Papilionoidea. En: Heppner, J.B. (Ed.). Atlas of Neotropical Lepidoptera. Vol. 5A. Association for Tropical Lepidoptera, Scientific Publishers, Gainesville, Florida, USA.

Llorente-Bousquets, J. 1983. Sinopsis sistemática y biogeográfica de los Dismorphiinae de México con especial referencia al género Enantia Hüebner (Lepidoptera: Pieridae). Folia Entomológica Mexicana, (58): 1-207.

Llorente-Bousquets, J. and P. Escalante-Pliego. 1992. Insular biogeography of submontane humid forests in Mexico. (pp. 139-146). In: Darwin, S.P. and A.L. Welden (Eds.). Biogeography of Mesoamerica. The E. O. Painter Printing. Florida, USA.

Llorente, J. y S. Ocegueda. 2008. Estado del conocimiento de la biota. (pp. 283-322). En: Soberón, J., G. Halffter y J. Llorente (Comps.) Volumen I. Conocimiento actual de la Biodiversidad. Capital Natural de México. (J. Sarukhán, Compilador Principal de la Obra). CONABIO. México, D. F.

Llorente-Bousquets, J., A. Luis-Martínez e I. Vargas Fernández. 2006. Apéndice general de Papilionoidea: Lista sistemática, distribución estatal y provincias biogeográficas. (pp. 945-1009). En: Morrone, J.J. y J. Llorente-Bousquets (Eds.). Componentes bióticos principales de la entomofauna mexicana, Vol. II. Las Prensas de Ciencias, UNAM, Ciudad de México, México, D. F.

Llorente-Bousquets, J., A. Luis-Martínez and A. Arellano-Covarrubias. 2013. A new subspecies of Synargis nymphidioides (Butler, 1872) (Lepidoptera, Riodini- 
dae): a prediction from a center of endemism in Sierra Madre del Sur, Mexico. Southwestern Entomologist, 38(4): 623-634.

Llorente-Bousquets, J., I. Vargas-Fernández, A. Luis-Martínez, M. Trujano-Ortega, B.C. Hernández-Mejía y A.D. Warren. 2014. Biodiversidad de Lepidoptera en México. Revista Mexicana de Biodiversidad, 85 (Supl.): S353-S371.

Luis-Martínez, A. y J. Llorente-Bousquets. 1990. Mariposas en el Valle de México: Introducción e Historia. 1. Distribución local y estacional de los Papilionoidea de la Cañada de los Dínamos, Magdalena Contreras, D. F., México. Folia Entomológica Mexicana, (78): 95198.

Luis-Martínez, A., I. Vargas-Fernández y J. Llorente-Bousquets. 1991. Lepidopterofauna de Oaxaca I: distribución y fenología de los Papilionoidea de la Sierra de Juárez. Publicaciones Especiales del Museo de Zoología, (3): 1-119.

Luis-Martínez, A., J. Llorente-Bousquets, A. Warren e I. Vargas-Fernández. 2004. Los lepidópteros: papilionoideos y hesperoideos. (pp. 331-356). En: García-Mendoza, A.J., M.J. Ordóñez y M.A. Briones-Salas (Eds.). Biodiversidad de Oaxaca. Instituto de Biología, UNAM-Fondo Oaxaqueño para la Conservación de la Naturaleza-WWF, México, D.F.

Luis-Martínez, A., J. Llorente-Bousquets e I. Vargas-Fernández. 2005. Una megabase de datos de mariposas de México y la regionalización biogeográfica. (pp. 269294). En: Llorente-Bousquets, J. y J.J. Morrone. (Eds.). Regionalización Biogeográfica en Iberoamérica y tópicos afines: Primeras Jornadas Biogeográficas RIBES. Las Prensas de Ciencias. México, D.F.

Luis-Martínez, A., M. Trujano, J. Llorente-Bousquets e I. Vargas-Fernández. 2006. Patrones de distribución de las subfamilias Danainae, Apaturinae, Biblidinae y Heliconiinae (Lepidoptera: Nymphalidae). (pp. 771-865). En: Morrone, J.J. y J. Llorente-Bousquets (Eds.). Componentes bióticos principales de la entomofauna mexica$n a$, Vol. II. Las Prensas de Ciencias, UNAM, Ciudad de México, México.

Luis-Martínez, A., A. Sánchez-García, O. Ávalos-Hernández, J.L. Salinas-Gutiérrez, M. Trujano-Ortega, A. Arellano-Covarrubias and J. Llorente-Bousquets. 2020. Distribution and diversity of Papilionidae and Pieridae (Lepidoptera: Papilionoidea) in Loxicha Region, Oaxaca, Mexico. Revista de Biología Tropical, 68(1): 139155.

Luis-Martínez, A., B.C. Hernández-Mejía, M. Trujano-Ortega, A. Warren, J. Salinas-Gutiérrez, O. Ávalos-Hernández, I. Vargas-Fernández y J. Llorente-Bousquets. 2016. Avances faunísticos en los Papilionoidea (Lepidoptera) sensu lato de Oaxaca, México. Southwestern Entomologist, 41(1): 171-224.

Monteagudo-Sabaté, D. y A. Luis-Martínez. 2013. Patrones de riqueza altitudinal de Papilionidae, Pieridae y Nymphalidae (Lepidoptera: Rhopalocera) en áreas monta- ñosas de México. Revista de Biología Tropical, 61(3): 1509-1520.

Monteagudo-Sabaté, D., A. Luis-Martínez, I. Vargas-Fernández y J. Llorente-Bousquets. 2001. Patrones altitudinales de diversidad de mariposas en la Sierra Madre del Sur (México) (Lepidoptera: Papilionoidea). SHILAP Revista de Lepidopterología, 29(115): 207-237.

Moreno, C.E. 2001. Métodos para medir la biodiversidad, Vol. 1. MyT-Manuales y Tesis SEA. Zaragoza, España.

Oñate-Ocaña, L., M. Trujano-Ortega, J. Llorente-Bousquets, A. Luis-Martínez e I. Vargas-Fernández. 2006. Patrones de distribución de la familia Papilionidae (Lepidoptera). (pp. 661-714). En: Morrone, J.J. y J. Llorente-Bousquets (Eds.). Componentes bióticos principales de la entomofauna mexicana, Vol. II. Las Prensas de Ciencias, UNAM. Ciudad de México, México

Pozo, C., A. Luis Martínez, N. Salas-Suárez, M. Trujano-Ortega y J. Llorente-Bousquets. 2015. Mariposas diurnas: bioindicadoras de eventos actuales e históricos. (pp. 327-348). En: González-Zuarth, C.A., A. Vallarino, J.C. Pérez-Jiménez y A.M. Low-Pfeng (Eds.). Bioindicadores: guardianes de nuestro futuro ambiental. ECOSUR-INECC. México.

Raguso, R.A. and J. Llorente. 1991. The Butterflies (Lepidoptera) of the Tuxtlas Mts., Veracruz, Mexico. Revisited: Species-Richness and Habitat Disturbance. Journal of Research on the Lepidoptera, 29 (1-2): 105-133.

Ross, G.N. 1975-1977. An ecological study of the butterflies of Sierra de Tuxtla, Veracruz, México. Journal of Research on the Lepidoptera, 14(2): 103-124; (3): 169188 ; (4): 233-252; 15(1): 41-60; (2): 109-128; (3): 185200; (4): 225-240; 16(2): 87-130.

Salinas-Gutiérrez, J.L., A. Luis-Martínez and J. LlorenteBousquets. 2004. Papilionoidea of the evergreen tropical forests of Mexico. Journal of the Lepidopterists' Society, 58 (3): 125-142.

Seraphim, N., L.A. Kaminski, P.J. Devries, C. Penz, C. Callaghan, N. Wahlberg, K.L. Silva-Brandao and A.V.L. Freitas. 2018. Molecular phylogeny and higher systematics of the metalmark butterflies (Lepidoptera: Riodinidae). Systematic Entomology, 43(2), 407-425.

Sparrow, H.R., T.D. Sisk, P.R. Ehrlich, and D.D. Murphy. 1994. Techniques and guidelines for monitoring neotropical butterflies. Conservation Biology, 8(3): 800809.

Sreekumar, P. and M. Balakrishnan. 2001. Habitat and altitude preference of butterflies in Aralam wildlife sanctuary, Kerala. Tropical Ecology, 42(2): 277-281.

Stanford, R.E. and P.A. Opler. 1993. Atlas of Western USA Butterflies Including Adjacent Parts of Canada and Mexico. self-published. Denver and Fort Collins, Colorado, USA.

Tangah, J., J. Hill, K. Hamer and M. Dawood. 2004. Vertical distribution of fruit-feeding butterflies in Sabah, Borneo. Sepilok Bulletin, 1(17): 17-27.

Torres-Rojo, J.M., O.S. Magaña-Torres y F. Moreno-Sán- 
chez. 2016. Predicción del cambio de uso/cobertura arbolada en México a través de probabilidades de transición. Agrociencia, 50(6): 769-758.

Trujano-Ortega, M., A. Luis-Martínez y J. Llorente-Bousquets. 2015. Variación morfológica y distribución de Theope villai (Lepidoptera: Riodinidae). Southwestern Entomologist, 40 (2): 333-350.

Trujano-Ortega, M., C. Callaghan, A. Arellano-Covarrubias, A. Luis-Martínez, O. Ávalos-Hernández and J. Llorente-Bousquets. 2021. Geographical distribution of Emesis Fabricius (Lepidoptera: Riodinidae) in Mexico: Updated checklist and temporal patterns. Zootaxa, 4964(3): 401-442.

Vargas-Fernández, I., J. Llorente-Bousquets y A. Luis-Martínez. 1994. Listado Lepidopterofaunístico de la Sierra de Atoyac de Álvarez en el estado de Guerrero: notas acerca de su distribución local y estacional (Rhopalocera: Papilionoidea). Folia Entomológica Mexicana, (86): 41-178.

Vargas-Fernández, I., J. Llorente-Bousquets y A. Luis-Mar-

Recibido: 5 mayo 2021

Aceptado: 4 junio 2021 tínez. 1999. Distribución de los Papilionoidea (Lepidoptera: Rhopalocera) de la Sierra de Manantlán (250-1,650 $\mathrm{m})$ en los estados de Jalisco y Colima. Publicaciones especiales del Museo de Zoología, (11): 1-153.

Vargas-Fernández, I., J. Llorente-Bousquets y A. Luis-Martínez. 2016. Adiciones a la serie Papilionoidea de México: distribución geográfica e ilustración. Las Prensas de Ciencias, UNAM. Ciudad de México, México.

Vargas-Fernández, I., M. Trujano, J. Llorente-Bousquets y A. Luis-Martínez. 2006. Patrones de distribución de las subfamilias Ithomiinae, Morphinae y Charaxinae (Lepidoptera: Nymphalidae). (pp. 867-943). En: Morrone, J.J. y J. Llorente Bousquets (Eds.). Componentes bióticos principales de la entomofauna mexicana, Vol. II. Las Prensas de Ciencias, UNAM, Ciudad de México, México.

Viloria, A. 2002. Limitaciones que ofrecen distintas interpretaciones taxonómicas y biogeográficas al inventario de lepidópteros hiperdiversos de las montañas Neotropicales y a sus posibles aplicaciones. $m 3 m$ : Monografías Tercer Milenio, 2: 173-190. Zaragoza, España.

Cuadro 1. Riqueza de especies y subespecies de cuatro familias de Papilionoidea en Oaxaca y la región Loxicha. La región presenta el $71 \%$ de los géneros, $20 \%$ de las especies y $49 \%$ de las subespecies del estado. Spp. $=$ especies; Sspp. $=$ subespecies.

\begin{tabular}{|c|c|c|c|c|c|c|c|}
\hline & & Oaxaca & & & Loxicha & & \\
\hline Familia & Subfamilias & Géneros & Spp. & Sspp. & Géneros & Spp. & Sspp. \\
\hline \multirow[t]{2}{*}{ Papilionidae } & Baroniinae & 1 & 1 & 1 & 1 & 1 & 1 \\
\hline & Papilioninae & 8 & 39 & 52 & 9 & 7 & 26 \\
\hline \multirow[t]{3}{*}{ Pieridae } & Dismorphiinae & 3 & 10 & 13 & 3 & 3 & 3 \\
\hline & Coliadinae & 12 & 27 & 28 & 11 & 8 & 23 \\
\hline & Pierinae & 16 & 24 & 27 & 11 & 1 & 16 \\
\hline \multirow[t]{11}{*}{ Nymphalidae } & Libytheinae & 1 & 1 & 1 & 1 & 1 & 1 \\
\hline & Danainae & 3 & 6 & 6 & 3 & 6 & 6 \\
\hline & Ithomiinae & 16 & 31 & 38 & 9 & 2 & 15 \\
\hline & Charaxinae & 10 & 40 & 47 & 10 & 4 & 21 \\
\hline & Morphinae & 6 & 17 & 21 & 3 & 3 & 8 \\
\hline & Satyrinae & 22 & 62 & 66 & 12 & 16 & 26 \\
\hline & Apaturinae & 2 & 6 & 6 & 2 & 3 & 3 \\
\hline & Biblidinae & 17 & 77 & 65 & 16 & 5 & 31 \\
\hline & Limenitidinae & 2 & 5 & 31 & 1 & 3 & 16 \\
\hline & Nymphalinae & 19 & 66 & 72 & 16 & 12 & 47 \\
\hline & Heliconiinae & 11 & 28 & 31 & 9 & 2 & 15 \\
\hline \multirow[t]{2}{*}{ Riodinidae } & Nemeobiinae & 2 & 11 & 13 & 1 & 2 & 2 \\
\hline & Riodininae & 44 & 111 & 117 & 23 & 31 & 50 \\
\hline TOTAL & & 195 & 562 & 635 & 141 & 110 & 310 \\
\hline
\end{tabular}


Cuadro 2. Comparación de la riqueza y endemismo de cuatro familias de Papilionoidea de dos regiones de la Sierra Madre del Sur en la vertiente del Pacífico. Ambas regiones se muestrearon de forma sistemática lo que permite la comparación de la riqueza.

Spp. = especies; Sspp. = subespecies. Riqueza/Endemismo.

\begin{tabular}{|c|c|c|c|c|c|c|c|}
\hline \multirow[b]{2}{*}{ Familia } & \multirow[b]{2}{*}{ Subfamilias } & \multicolumn{3}{|c|}{ región Loxicha } & \multicolumn{3}{|c|}{ Sierra de Atoyac } \\
\hline & & Géneros & Spp. & Sspp. & Géneros & Spp. & Sspp. \\
\hline \multirow[t]{2}{*}{ Papilionidae } & Baroniinae & $1 / 1$ & 1 & $1 / 1$ & - & - & - \\
\hline & Papilioninae & 9 & $7 / 3$ & $26 / 9$ & 8 & $7 / 3$ & $20 / 8$ \\
\hline \multirow[t]{3}{*}{ Pieridae } & Dismorphiinae & 3 & 3 & $3 / 3$ & 3 & $1 / 1$ & $4 / 3$ \\
\hline & Coliadinae & $11 / 1$ & $8 / 1$ & 23 & 9 & 5 & 20 \\
\hline & Pierinae & $11 / 1$ & $1 / 1$ & $16 / 3$ & 10 & 13 & $13 / 2$ \\
\hline \multirow[t]{11}{*}{ Nymphalidae } & Libytheinae & 1 & 1 & 1 & 1 & 1 & 1 \\
\hline & Danainae & 3 & 6 & 6 & 3 & 5 & 5 \\
\hline & Ithomiinae & 9 & $2 / 1$ & $15 / 5$ & 7 & $2 / 1$ & $12 / 7$ \\
\hline & Charaxinae & 10 & $4 / 1$ & $21 / 7$ & 9 & 3 & $16 / 6$ \\
\hline & Morphinae & 3 & 3 & $8 / 1$ & 3 & 2 & $7 / 1$ \\
\hline & Satyrinae & 12 & $16 / 5$ & $26 / 7$ & 12 & $20 / 7$ & $35 / 7$ \\
\hline & Apaturinae & 2 & 3 & 3 & 1 & 1 & 1 \\
\hline & Biblidinae & 16 & $5 / 1$ & $31 / 14$ & 14 & $6 / 1$ & $29 / 13$ \\
\hline & Limenitidinae & 1 & 3 & $16 / 3$ & 1 & 2 & $13 / 2$ \\
\hline & Nymphalinae & 16 & $12 / 2$ & $47 / 6$ & 15 & $12 / 1$ & $35 / 4$ \\
\hline & Heliconiinae & 9 & 2 & $15 / 3$ & 7 & 19 & $12 / 3$ \\
\hline \multirow[t]{2}{*}{ Riodinidae } & Nemeobiinae & 1 & 2 & 2 & 1 & 2 & 2 \\
\hline & Riodininae & $23 / 3$ & $31 / 6$ & $50 / 3$ & 10 & $21 / 3$ & $35 / 2$ \\
\hline TOTAL & & $141 / 6$ & 110 / 21 & $310 / 65$ & 114 & $122 / 17$ & $260 / 58$ \\
\hline
\end{tabular}

